\title{
Simulation and experimental study on PID control of a quadrotor MAV with perturbation
}

\author{
A. Noordin ${ }^{1}$, M. A. M. Basri ${ }^{2}$, Z. Mohamed ${ }^{3}$ \\ ${ }^{1,2,3}$ School of Electrical Engineering, Universiti Teknologi Malaysia, Malaysia \\ ${ }^{1}$ Faculty of Electrical and Electronic Engineering Technology, Universiti Teknikal Malaysia Melaka, Malaysia
}

\begin{abstract}
Article Info
Article history:

Received Jan 29, 2020

Revised Mar 8, 2020

Accepted Apr 9, 2020

\section{Keywords:}

Altitude control External disturbance PID

Position control

Quadrotor MAV

ABSTRACT

This paper presents a proportional-integral-derivative (PID) flight controller for a quadrotor micro air vehicle (MAV). The MAV (Parrot Mambo minidrones) is small, therefore, a slight perturbation will affect its performance. Hence, for the actuated dynamics, roll $(\phi)$, pitch $(\theta)$, yaw $(\psi)$, and $\mathrm{z}$ stabilization, a PID control scheme is proposed. Furthermore, the same controller technique is also applied for under-actuated dynamics $\mathrm{x}$ and $\mathrm{y}$ position control. The newtonian model is simulated using simulink with a normal Gaussian noise of force as external disturbances. using simulink support package for Parrot Minidrones by MATLAB and based on the simulation parameter, the algorithm is deployed using Bluetooth ${ }^{\circledR}$ Low energy connection via personal area network (PAN). A slight force by hand is applied as perturbation during hovering to investigation system performances. Finally, the simulation and experimental on this commercial MAV, Parrot Mambo minidrones shows good performance of the flight controller scheme in the presence of external disturbances.
\end{abstract}

This is an open access article under the CC BY-SA license.

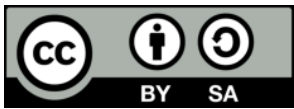

\section{Corresponding Author:}

M. A. M. Basri,

School of Electrical Engineering,

Universiti Teknologi Malaysia,

81310 Skudai, Johor Bahru, Johor, Malaysia.

Email: ariffanan@fke.utm.my

\section{INTRODUCTION}

Recently, many quadrotor drones have been developed based on their size and purposes such as mini drones, hobby drones, professional drones, selfie drones, and FPV racing drone [1]. Mini drone or micro air vehicle (MAV) defined as a small scale UAV with mass $<0.1 \mathrm{~kg}$ get attention by educator/researcher due to it reliable and safe to perform in denied GPS space such as in the halls, schools, and more [2].

DJI and Parrot are among the well-known manufacturers that have established and commercialized quadrotor drones which cover most of those types including for educations. For instance, DJI developed Tello EDU, which can be programmed through Scratch 2.0, SDK 2.0, and even support Swift Playgrounds for iOS user [3]. In a while, Parrot has developed AR Drone 2.0, Rolling Spider and Mambo with all model support to be programmed via MATLAB/Simulink. AR Drone 2.0 toolbox is developed and shared by researchers while Simulink Support Package for Rolling Spider which recently upgraded to Simulink Support Package for Parrot Minidrones is add-on hardware support packaged provided by MATLAB based on Aerospace Blockset developed by MIT [4].

The mini drone's quadrotor structure is simple, yet under-actuated and dynamically unstable system makes it complicated to design a full controller. Therefore, most of the research used common techniques 
by creating two virtual inputs; roll angle and pitch angle for position control which required a very stabilized attitude controller [5-7]. Various control method has been presented such as an backstepping control [8-12], feedback linearization linear control [13, 14], sliding mode control (SMC) for attitude and altitude [15-19], proportional-integral-derivative (PID) [20-23] and LQR control [24, 25] and fuzzy logic control [26, 27].

This paper presents a PID control for under-actuated Parrot Mambo mini-drone focus on attitude stabilization during position control. The advantages of PID is its practicability and easy to be implemented just based on the system tracking error [28, 29]. The Parrot Mambo (6-DOF quadrotor mini drone), includes with ultrasonic, accelerometer, gyroscope, air pressure and down-facing camera sensors. It allows Bluetooth ${ }^{\circledR}$ Low Energy connection to deploy algorithms wirelessly via personal area network (PAN). This paper contributes a demonstration of simulation and experimental results of the PID controller applied to a commercial MAV (Parrot Mambo minidrone).

This paper is organized as follows. Section 2 describes the nonlinear dynamic model based on the Parrot Mambo, and the design of the PID for both attitude and position control of the quadrotor. Section 3 presents the simulation and experiment results of controllers. Section IV is the conclusion of the paper.

\section{RESEARCH METHOD}

In this section, the nonlinear dynamics model of the Parrot Mambo mini-drone is presented using Newton-Euler formulation.

\subsection{Quadrotor dynamics}

In (1) [30] describes the translational dynamic model, while (2) represents the rotational dynamic model for $\times$-model of the Parrot Mambo mini-drone, as shown in Figure 1.

$$
\begin{aligned}
& \ddot{x}=\frac{1}{m} U_{1}\left(S \psi S \phi+C \psi S \theta C \phi_{x}\right) \\
& \ddot{y}=\frac{1}{m} U_{1}(-C \psi S \phi+S \psi S \theta C \phi) \\
& \ddot{z}=g+\frac{1}{m}(C \theta C \phi) U_{1} \\
& \frac{\left(I_{y y}-I_{z z}\right)}{I_{x x}} \dot{\psi} \dot{\theta}-\frac{J_{r}}{I_{x x}}\left(\Omega_{d}\right) \dot{\theta}+\frac{l}{I_{x x}} U_{2} \\
& \ddot{\theta}=\frac{\left(I_{z z}-I_{x x}\right)}{I_{y y}} \dot{\psi} \dot{\phi}+\frac{J_{r}}{I_{y y}}\left(\Omega_{d}\right) \dot{\phi}+\frac{l}{I_{y y}} U_{3} \\
& \ddot{\psi}=\frac{\left(I_{x x}-I_{y y}\right)}{I_{z z}} \dot{\theta} \dot{\phi}+\frac{1}{I_{z z}} U_{4}
\end{aligned}
$$

where $S$ and $C$ stand for trigonometric operator 'sin' and 'cos' respectively, $g$ is gravitational coefficient, $m$ is quadrotor mass, $\phi, \theta, \psi$ represent roll, pitch, and yaw respectively. $I_{x x}, I_{y y}$, and $I_{z z}$ is the total moment of inertia, $J_{r}$ is the rotor moment of inertia, $\Omega_{d}$ is the total of angular speed, $U_{1}, U_{2}, U_{3}$, and $U_{4}$ are the control inputs. Table 1 shows the physical parameters of the Parrot Mambo, which is written in the source code obtained from the compilation of simulation model.

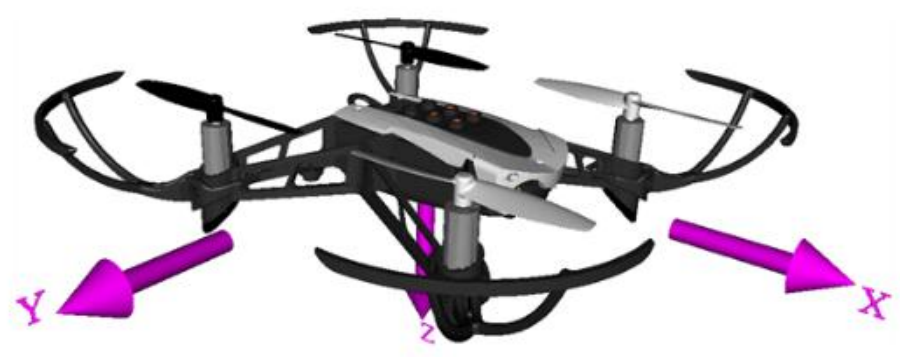

Figure 1. Parrot Mambo minidrone 
Table 1. Parrot Mambo model physical parameters

\begin{tabular}{cccc}
\hline Specification & Parameter & Unit & Value \\
\hline Quadrotor mass & $m$ & $\mathrm{~kg}$ & 0.063 \\
Lateral moment arm & $l$ & $\mathrm{~m}$ & 0.0624 \\
Thrust coefficient & $b$ & $\mathrm{Ns}^{2}$ & 0.0107 \\
Drag coefficient & $d$ & $\mathrm{Nms}^{2}$ & $0.78264 \times 10^{-3}$ \\
Rolling moment of inertia & $I_{x x}$ & $\mathrm{kgm}^{2}$ & 0.0000582857 \\
Pitching moment of inertia & $I_{y y}$ & $\mathrm{kgm}^{2}$ & 0.0000716914 \\
Yawing moment of inertia & $I_{z z}$ & $\mathrm{kgm}^{2}$ & 0.0001 \\
Rotor moment of inertia & $J_{r}$ & $\mathrm{kgm}^{2}$ & $0.1021 \times 10^{-6}$ \\
\hline
\end{tabular}

\subsection{Attitude controller}

The altitude controller is designed using classical PID as:

$$
U_{i}=k_{P}^{i} e_{i}+k_{I}^{i} \int e_{i} d t+k_{D}^{i} \dot{e}_{i}(i=\phi, \theta, \psi)
$$

where $e_{i}=i_{d}-i$ and $\dot{e}_{i}=\dot{i}_{d}-\dot{i}$ are the error and derivative error between the desired signal and actual signal, and $k_{P}^{i}, k_{I}^{i}$, and $k_{D}^{i}$ are the PID gains parameter $(i=\phi, \theta, \psi)$.

\subsection{Altitude controller}

The altitude controller is designed using classical PID with a gravity compensator controller and described as:

$$
U_{1}=k_{P}^{Z} e_{z}+k_{I}^{z} \int e_{z}+k_{D}^{z} \dot{e}_{z}+m g
$$

where $e_{z}=z_{d}-z$ and $\dot{e}_{z}=\dot{z}_{d}-\dot{z}$ are the error and derivative error between the desired signal and actual signal, and $k_{P}^{Z}, k_{I}^{Z}$, and $k_{D}^{Z}$ are the PID gain parameter.

\subsection{Position controller}

For the position control, since the MAV is operating around hover, the small-angle assumption $\left(S \phi_{d} \equiv \phi_{d}, S \theta_{d} \equiv \theta_{d}\right.$ and $\left.C \phi_{d}=C \theta_{d}=1\right)$ is applied to simplify (1) as:

$$
\begin{aligned}
& \ddot{x}=\frac{1}{m} U_{1}\left(\phi_{d} S \psi+\theta_{d} C \psi\right) \\
& \ddot{y}=\frac{1}{m} U_{1}\left(-\phi_{d} C \psi+\theta_{d} S \psi\right)
\end{aligned}
$$

Therefore, from (4), a virtual control signal is proposed to control the $\mathrm{x}$ and $\mathrm{y}$ position and can be implemented as:

$$
\begin{aligned}
& \phi_{d}=\left(u_{x} S \psi-u_{y} C \psi\right) \\
& \theta_{d}=\left(u_{x} C \psi+u_{y} S \psi\right)
\end{aligned}
$$

where $u_{x}$ and $u_{y}$ are the inputs control signal designed using PID as:

$$
\begin{aligned}
& u_{x}=k_{P}^{x} e_{x}+k_{I}^{x} \int e_{x}+k_{D}^{x} \dot{e}_{x} \\
& u_{y}=k_{P}^{y} e_{y}+k_{I}^{y} \int e_{y}+k_{D}^{y} \dot{e}_{y}
\end{aligned}
$$

\section{RESULTS AND DISCUSSION}

In this section, simulation and real-time implementation are carried out to verify the performance of the Parrot Mambo mini drone.

\subsection{Simulation results}

Based on the dynamics of quadrotor MAV in $(1,2)$ and PID controller in $(3,4,7)$, the simulations were conducted through MATLAB/Simulink ${ }^{\circledR}$ R2019a by setting third-order solver with 0.005 second sampling period. The dynamics $z, \phi, \theta$, and $\psi$ were evaluated with the presences of external disturbances, a normal Gaussian noise of force defined as $d(t)=[(0,0.5) N,(0,0.5) N,(0,0.5) N,(0,0.5) N]^{T}$ at the time interval $d(t)>10 s$ [15]. Figure 2(a) shows the altitude and the attitude response of the PID controller with 
a with the presence of external disturbance. As shown, the PID controller able to converge to zero even with the presence of external disturbances after $10 \mathrm{~s}$. Figure 2(b) shows the controller control inputs were sensitive to the noise after 10 seconds period.
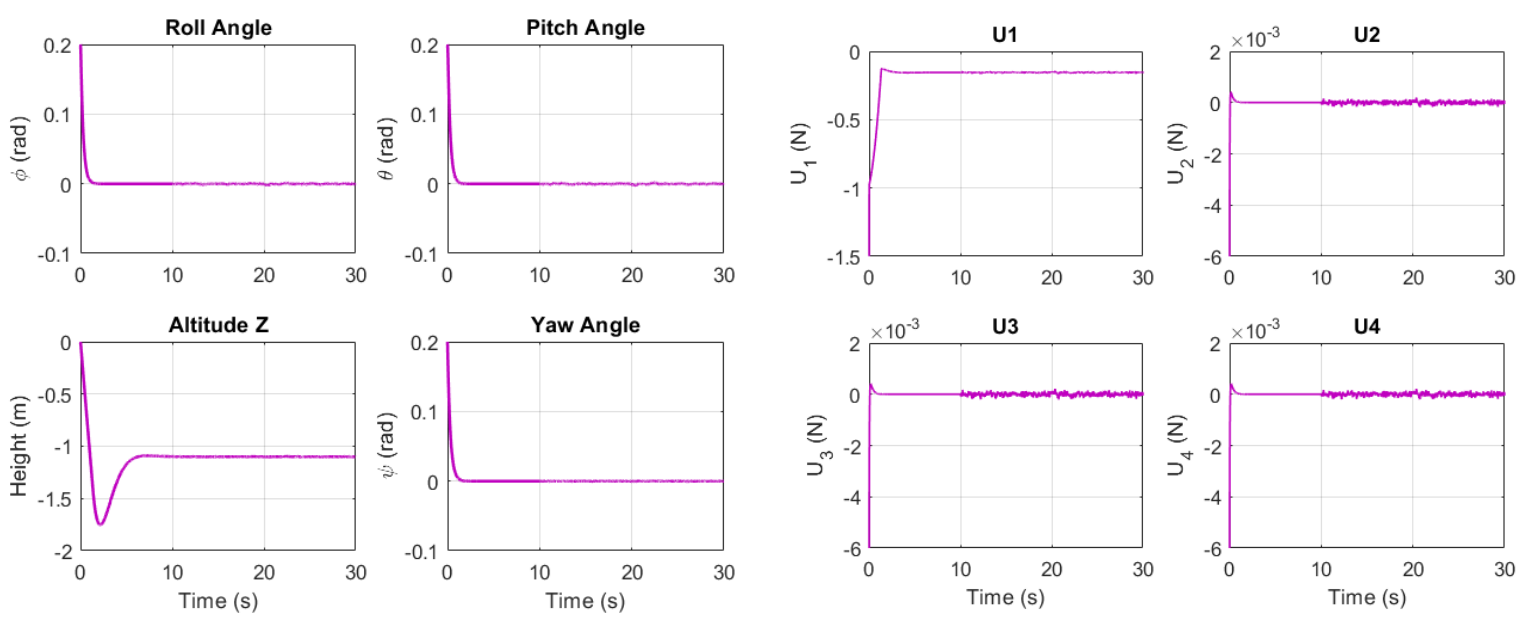

(a)

(b)

Figure 2. PID controller with the presence of external disturbance, (a) Altitude and attitude response,

(b) The control inputs

\subsection{Experimental results}

By using the Simulink Support Package for Parrot minidrones, the Parrot Mambo algorithm is deployed via Bluetooth. The state estimation was predefined in the provided package. The sensor fusion algorithm was designed using a complementary filter and Kalman filter to process onboard sensors such as the ultrasonic sensor, IMU, air pressure sensor, and optical flow sensor [2]. Figure 3 shows an experimental setup for MAV using Simulink for simulation and straight away can be programmed wirelessly through Bluetooth for real-time implementation.

The information from the real-time experiment is obtained from built-in memory in Parrot Mambo mini drone which is designed to log essential data such as sensors, positions (x,y, z) and rotational (roll, pitch, yaw). Initially, the desired altitude is set at $z=-1.1$ meter, while for the rotational angle yaw is set as $\psi=0$. Table 2 shows the PID parameters used in this experiment.

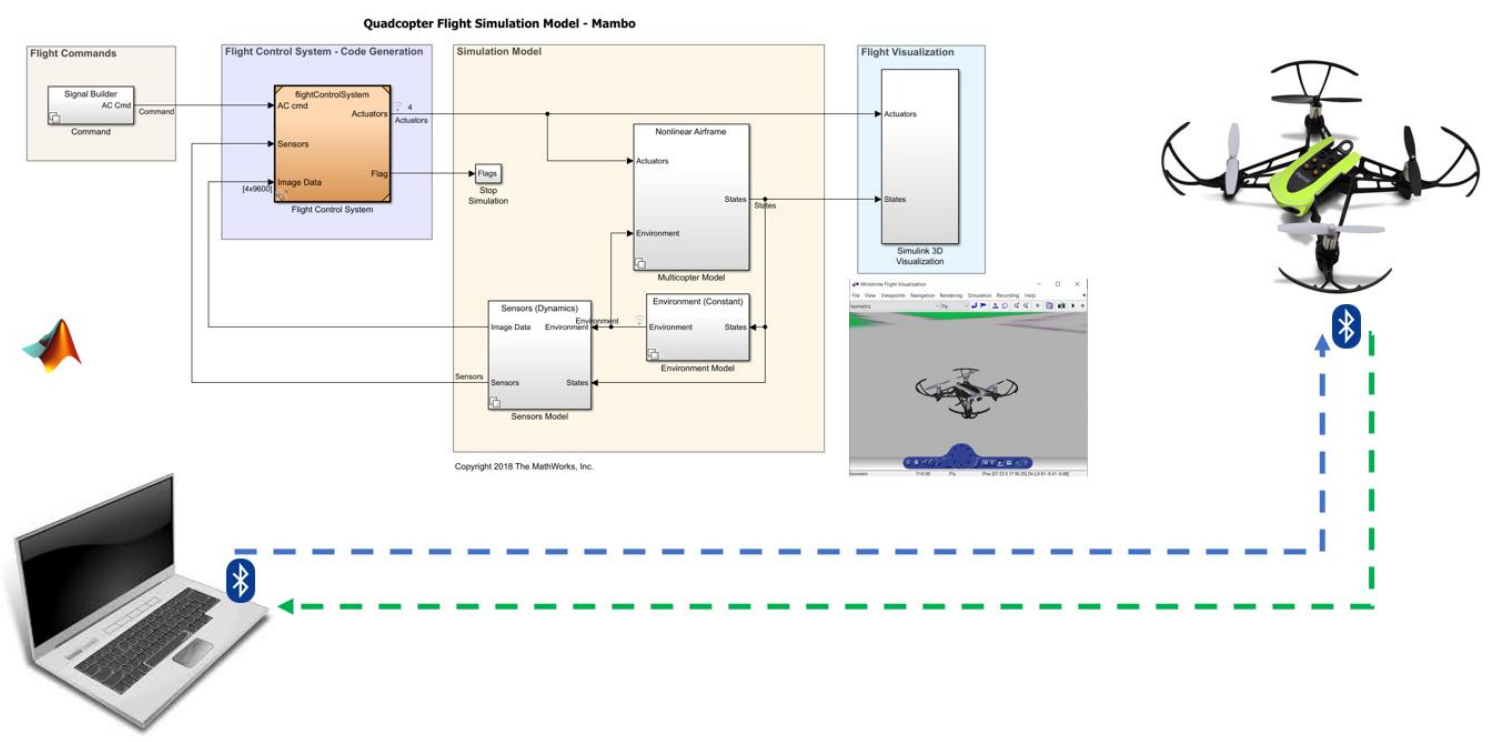

Figure 3. Experimental setup for MAV 
Table 2. PID parameters the Parrot Mambo controller

\begin{tabular}{cccc}
\hline & $\mathrm{Kp}$ & $\mathrm{Ki}$ & $\mathrm{Kd}$ \\
\hline Roll, $\phi$ & 0.011 & 0.01 & 0.0028 \\
Pitch, $\theta$ & 0.013 & 0.01 & 0.002 \\
Yaw, $\psi$ & 0.004 & 0.02 & 0.012 \\
$\mathrm{Z}$ & 0.800 & 0.24 & 0.500 \\
$\mathrm{Y}$ & -0.2 & 0.002 & 0.1 \\
$\mathrm{X}$ & 0.2 & 0.05 & -0.1 \\
\hline
\end{tabular}

\subsubsection{Hovering (altitude tracking without perturbations)}

In Figure 4(a), altitude $\mathrm{z}$ can reach $1.1 \mathrm{~m}$ as the desired with slightly oscillations contra to the simulation (nominal model). The system performances are affected due to parametric uncertainties, i.e., hull is included for safety, which increased the weight and inertia moments, and noise from onboard sensors. The roll angle and pitch angle capable of following the desired response from the virtual controller, and yaw angle were forced to converge to zero. Figure 4(b) shows motors angular velocities during hovering.
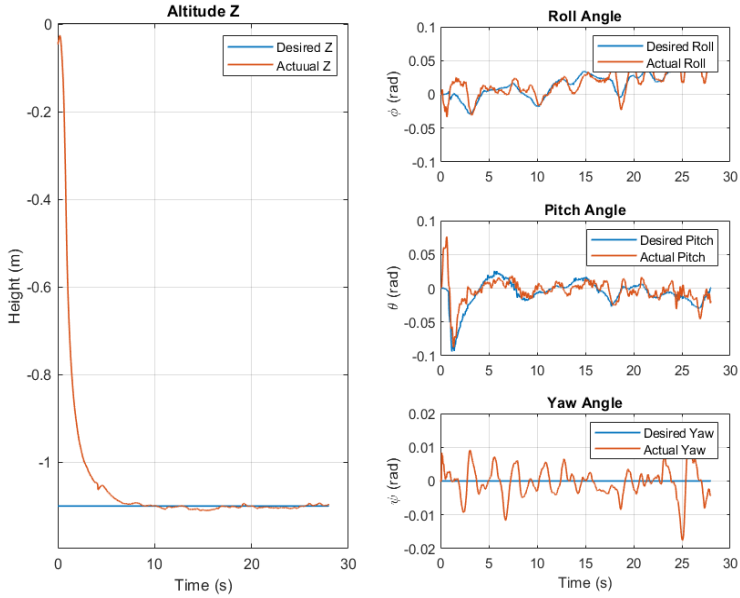

(a)
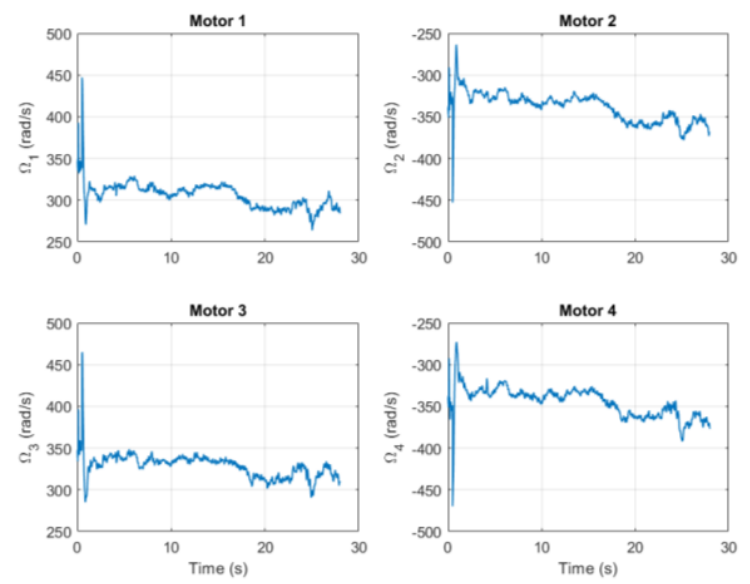

(b)

Figure 4. Parrot Mambo minidrone in hover position, (a) Altitude and attitude responses,

(b) Motors angular velocities

\subsubsection{Hovering (altitude tracking with perturbations)}

In this experiment, an external disturbance consist of a force by hand is applied twice to the quadrotor at $10 \mathrm{~s}$ and $15 \mathrm{~s}$ during hovering. Since the Parrot Mambo is small MAV, a slight perturbation will affect its performances. Figure 5(a) shows the output response of the system subject to an external perturbation. Figure 5(b) shows motors angular velocities during hovering subject to an external perturbation.

\subsubsection{Position tracking}

In this experiment, the initial position of the Parrot Mambo mini-drone is set as $[x, y, z]^{T}=$ $[0,0,0]^{T} m$ and the desired final position of the Parrot Mambo minidrone is set to $[x, y, z]^{T}=[1,1,-1.1]^{T} m$. In order to accomplish this, a Stateflow bock is used to design the path planning. On the beginning, the Parrot Mambo minidrone is set to hover by setting the output as $[x, y, z]^{T}=[0,0,-1.1]^{T} m$. After 4 seconds, the MAV is set to move forward by $[x, y, z]^{T}=[1,0,-1.1]^{T} m$ and then after 15 seconds the MAV is set to move to the right side by $[x, y, z]^{T}=[1,1,-1.1]^{T} \mathrm{~m}$, and finally hover at that condition afterward. Figure 6(a) shows the output response of the Parrot Mambo minidrone to follow the path as planned. From the figure, trajectory $x$ and $y$ is forced to converge to dedicated output with slightly oscillation due to parameter uncertainties as mention above. Nevertheless, the output response of the attitude $\phi, \theta, \psi$, and altitude $z$ shows great stability in following the desired signal. Figure 6(b) shows motors angular velocities during position tracking. 

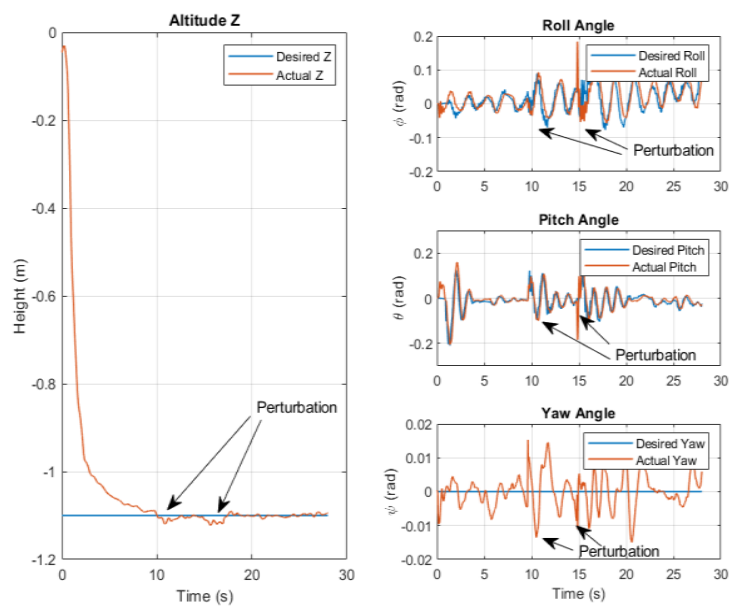

(a)


(b)

Figure 5. Parrot Mambo minidrone in hover position under external perturbations, (a) Altitude and attitude responses, (b) Motors angular velocities
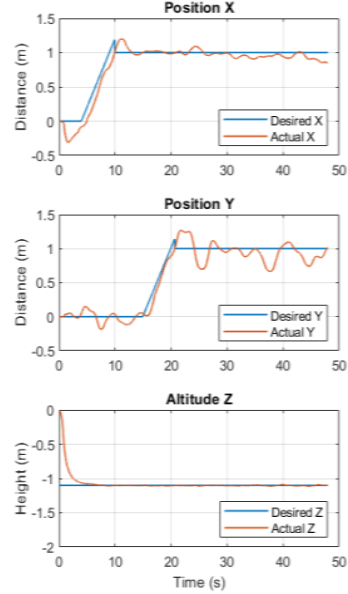

(a)
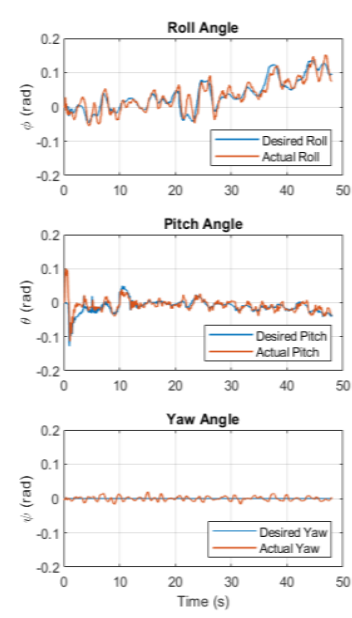
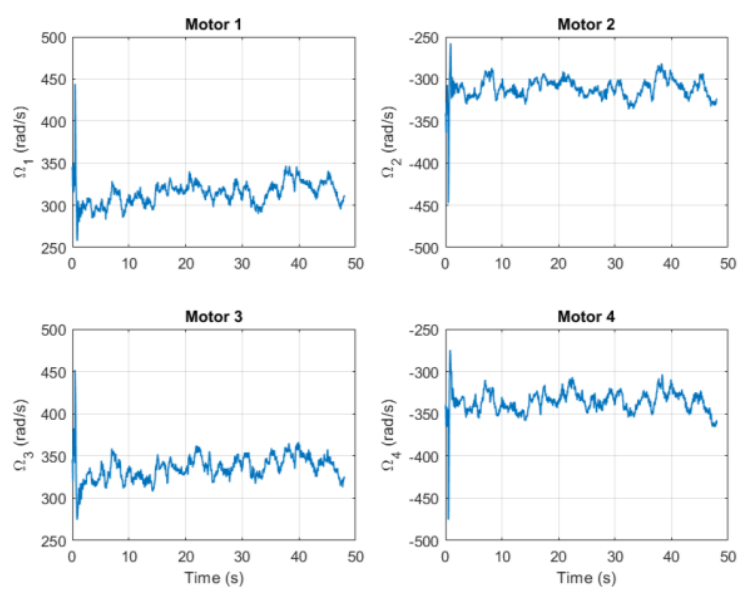

(b)

Figure 6. Parrot Mambo mini-drone position tracking,(a) Position and Attitude responses,

(b) Motors angular velocities

\section{CONCLUSION}

This paper presents a position and an attitude control implemented on the commercial MAV. For the attitude control, a PID control was proposed to stabilize the MAV subjected to small perturbation. Furthermore, for the position control, a PID controller with gravity compensator was utilized to ensure quadrotor able to manoeuvre successfully to the desired position by following the path as planned. Finally, in the conducted simulations and experiments on the Parrot Mambo minidrone with small perturbations, the PID controller demonstrates the MAV can achieve attitude stabilization, hover at desired altitude and then follow the desired trajectory as planned. For future work, a robust controller such as the adaptive PID or the sliding mode control techniques can be simulated and implemented on this commercial MAV

\section{ACKNOWLEDGEMENTS}

The authors would like to thank Universiti Teknologi Malaysia (UTM) under the Research University Grant (R.J130000.2651.17J42), Universiti Teknikal Malaysia Melaka (UTeM), and Ministry of Education Malaysia for supporting this research. 


\section{REFERENCES}

[1] Air Drone Craze, "5 Different Types of Drones for Consumers,". [Online]. Available: www.airdronecraze.com. [Accessed: 10-Jul-2019].

[2] H. Castañeda and J. L. Gordillo, "Embedded Flight Control Based on Adaptive Sliding Mode Strategy for a Quadrotor Micro Air Vehicle," Electronics, vol. 8, no. 7, 2019.

[3] Ryze Technology, "Tello EDU Drone,". [Online]. Available: https://www.ryzerobotics.com/. [Accessed: 10-Jul-2019].

[4] MathWorks, "Parrot Minidrones Support from Simulink,". [Online]. Available: https://www.mathworks.com/hardware-support/parrot-minidrones.html. [Accessed: 10-Jul-2019].

[5] Alia F. Abdul Ghaffar and Thomas S. Richardson, "Position Tracking of an Underactuated Quadrotor using Model Reference Adaptive Control," AIAA Guidance, Navigation, and Control Conference, pp. 1-13, 2016.

[6] H. J. Jayakrishnan, "Position and Attitude Control of A Quadrotor UAV Using Super Twisting Sliding Mode," IFAC-PapersOnLine, vol. 49, no. 1, pp. 284-289, 2016.

[7] W. Dong, G.-Y. Gu, X. Zhu, and H. Ding, "High-Performance Trajectory Tracking Control of A Quadrotor with Disturbance Observer," Sensors and Actuators A: Physical, vol. 211, pp. 67-77, May 2014.

[8] M. A. Mohd Basri, A. R. Husain, and K. A. Danapalasingam, "Intelligent Adaptive Backstepping Control for MIMO Uncertain Non-Linear Quadrotor Helicopter Systems," Transactions of the Institute of Measurement and Control, vol. 37, no. 3, pp. 345-361, 2015.

[9] M. Bouchoucha, S. Seghour, H. Osmani, and M. Bouri, "Integral Backstepping for Attitude Tracking of A Quadrotor System," Elektron. ir Elektrotechnika, vol. 116, no. 10, pp. 75-80, 2011.

[10] H. Khebbache and M. Tadjine, "Robust Fuzzy Backstepping Sliding Mode Controller for A Quadrotor," Journal of Control Engineering and Applied Informatics, vol. 15, no. 2, pp. 3-11, 2013.

[11] Yu Yali, Jiang Changhong and Wu Haiwei, "Backstepping control of each channel for a quadrotor aerial robot," 2010 International Conference on Computer, Mechatronics, Control and Electronic Engineering, Changchun, pp. 403-407, 2010.

[12] N. Xuan-mung, "Robust Backstepping Trajectory Tracking Control of a Quadrotor with Input Saturation via Extended State Observer," Applied Sciences, vol. 9, no. 23, 2019.

[13] I. M. Lazim, A. Rashid Husain, N. Adilla Mohd Subha, and M. Ariffanan Mohd Basri, "Intelligent Observer-Based Feedback Linearization for Autonomous Quadrotor Control," International Journal of Engineering \& Technology, vol. 7, no. 4.35, pp. 904-911, 2018.

[14] I. M. Lazim, A. R. Husain, M. Ariffanan, M. Basri, and N. A. Mohd, "Feedback Linearization with Intelligent Disturbance Observer for Autonomous Quadrotor with Time-varying Disturbance," International Journal of Mechanical \& Mechatronics Engineering IJMME-IJENS, vol. 18, no. 5, pp. 47-55, October 2018.

[15] A. Noordin, M. A. M. Basri, and Z. Mohamed, "Sliding Mode Control for Altitude and Attitude Stabilization of Quadrotor UAV with External Disturbance," Indonesian Journal of Electrical Engineering and Informatics (IJEEI), vol. 7, no. 2, pp. 203-210, 2019.

[16] S. Bouabdallah and R. Siegwart, "Backstepping and Sliding-mode Techniques Applied to an Indoor Micro Quadrotor," Proceedings of the 2005 IEEE International Conference on Robotics and Automation, Barcelona, Spain, pp. 2247-2252, 2005.

[17] H. Bouadi and M. Tadjine, "Nonlinear Observer Design and Sliding Mode Control of Four Rotors Helicopter," Proceedings Of World Academy Of Science, Engineering And Technology, vol. 25, no. 7, pp. 225-230, 2007.

[18] G. Perozzi, D. Efimov, J. M. Biannic, and L. Planckaert, "Trajectory Tracking for A Quadrotor Under Wind Perturbations: Sliding Mode Control with State-Dependent Gains," Journal of the Franklin Institute, vol. 355, no. 12, pp. 4809-4838, 2018.

[19] S. Riache, M. Kidouche, A. Rezoug, "Adaptive Robust Nonsingular Terminal Sliding Mode Design Controller for Quadrotor Aerial Manipulator," TELKOMNIKA Telecommunication Comput. Electron. Control, vol. 17, no. 3, pp. 1501-1512, 2019.

[20] A. R. Al Tahtawi, M. Yusuf, "Low-Cost Quadrotor Hardware Design with PID Control System As Flight Controller," TELKOMNIKA Telecommunication Comput. Electron. Control, vol. 17, no. 4, pp. 1923-1930, 2019.

[21] M. Nguyen Duc, T. N. Trong and Y. S. Xuan, "The quadrotor MAV system using PID control," 2015 IEEE International Conference on Mechatronics and Automation (ICMA), Beijing, pp. 506-510, 2015.

[22] S. J. Haddadi, O. Emamagholi, F. Javidi and A. Fakharian, "Attitude control and trajectory tracking of an autonomous miniature aerial vehicle," 2015 AI \& Robotics (IRANOPEN), Qazvin, pp. 1-6, 2015.

[23] G. Szafranski, R. Czyba, "Different Approaches of PID Control UAV Type Quadrotor," Proceedings of the International Micro Air Vehicles conference 2011 summer edition., pp. 70-75, 2011.

[24] Z. Shulong, A. Honglei, Z. Daibing and S. Lincheng, "A new feedback linearization LQR control for attitude of quadrotor," 2014 13th International Conference on Control Automation Robotics \& Vision (ICARCV), Singapore, pp. 1593-1597, 2014.

[25] S. Khatoon, D. Gupta and L. K. Das, "PID \& LQR control for a quadrotor: Modeling and simulation," 2014 International Conference on Advances in Computing, Communications and Informatics (ICACCI), New Delhi, pp. 796-802, 2014.

[26] J. O. Pedro and P. J. Kala, "Nonlinear control of quadrotor UAV using Takagi-Sugeno fuzzy logic technique," 2015 10th Asian Control Conference (ASCC), Kota Kinabalu, pp. 1-6, 2015.

[27] H. Wicaksono, Y. G. Yusuf, C. Kristanto, and L. Haryanto, "Outdoor Altitude Stabilization of Quadrotor Based on Type-2 Fuzzy and Fuzzy PID," IOP Conference Series: Materials Science and Engineering, International Conference on Informatics, Technology and Engineering 2017, Indonesia, vol. 273, no. 1, 2017. 
[28] B. R. Yenugula and M. Ziz-ur-Rahman, "Stability Control Structure of Hovercraft Prototype Utilising PID Controller," Bulletin of Electrical Engineering and Informatics, vol. 6, no. 4, pp. 348-350, 2017.

[29] C. Sharma and A. Jain, "Basis Weight Gain Tuning Using Different Types of Conventional Controllers," Bulletin of Electrical Engineering and Informatics, vol. 5, no. 1, pp. 62-71, 2016.

[30] A. Noordin, M. A. M. Basri, Z. Mohamed, and A. F. Z. Abidin, "Modelling and PSO Fine-Tuned PID Control of Quadrotor UAV," International Journal on Advanced Science, Engineering and Information Technology, vol. 7, no. 4, pp. 1367-1373, 2017.

\section{BIOGRAPHIES OF AUTHORS}

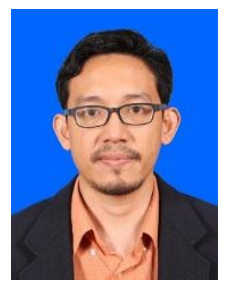

Aminurrashid Noordin received the B.Eng. and the M.Eng. Degree in Mechatronics Engineering from Universiti Teknologi Malaysia in 2002 and 2009 respectively, where he is currently working toward the Ph.D. in the Department of Control and Mechatronics Engineering of Universiti Teknologi Malaysia (UTM). Since 2011, he has been with Department of Electrical Engineering Technology, Faculty of Electrical and Electronic Engineering Technology of Universiti Teknikal Malaysia Melaka where he is currently a Senior Lecturer. His research interests include Nonlinear Control System, Robotics and Embedded System.



Mohd Ariffanan Mohd Basri received the B.Eng. and the M.Eng. Degree in Mechatronics Engineering from Universiti Teknologi Malaysia in 2004 and 2009 respectively. He also received the Ph.D. in Electrical Engineering from Universiti Teknologi Malaysia in 2015. He is currently a Senior Lecturer in Department of Control and Mechatronics Engineering of Universiti Teknologi Malaysia. His research interests include intelligent and Nonlinear Control Systems.

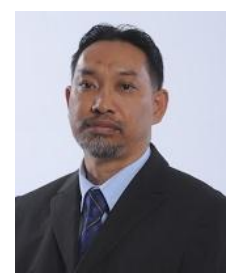

Zaharuddin Mohamed received his B.Eng. in Electrical, Electronics and Systems from Universiti Kebangsaan Malaysia (UKM) in 1993, M.Sc. in Control Systems Engineering from The University of Sheffield in 1995 and Ph.D. in Control Systems Engineering from The University of Sheffield in 2003. Currently, he is a Professor in Department of Control and Mechatronics Engineering of Universiti Teknologi Malaysia (UTM) and his current research interest involve the control of Mechatronics systems, flexible and smart structures. 